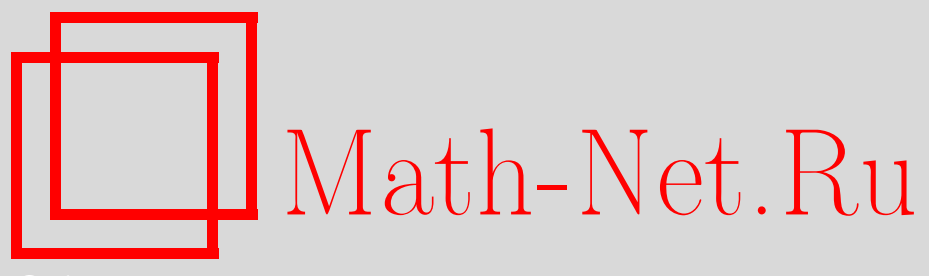

А. М. Абрамов, В. И. Арнольд, А. В. Болсинов, А. Н. Варченко, Л. Гальгани, Б. И. Жилинский, Ю. С. Ильяшенко, В. В. Козлов, А. И. Нейштадт, В. И. Питербарг, А. Г. Хованский, В. В. Ященко, Николай Николаевич Нехорошев (некролог), УМH, 2009, том 64, выпуск 3, 174-178

DOI: https://doi.org/10.4213/rm9288

Использование Общероссийского математического портала Math-Net.Ru подразумевает, что вы прочитали и согласны с пользовательским соглашением http://www . mathnet.ru/rus/agreement

Параметры загрузки:

IP: 54.164 .48 .24

26 апреля 2023 г., 03:39:49

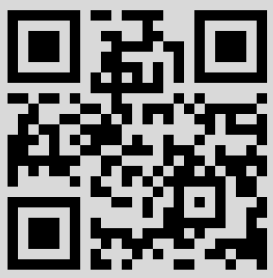




\section{Николай Николаевич Нехорошев}

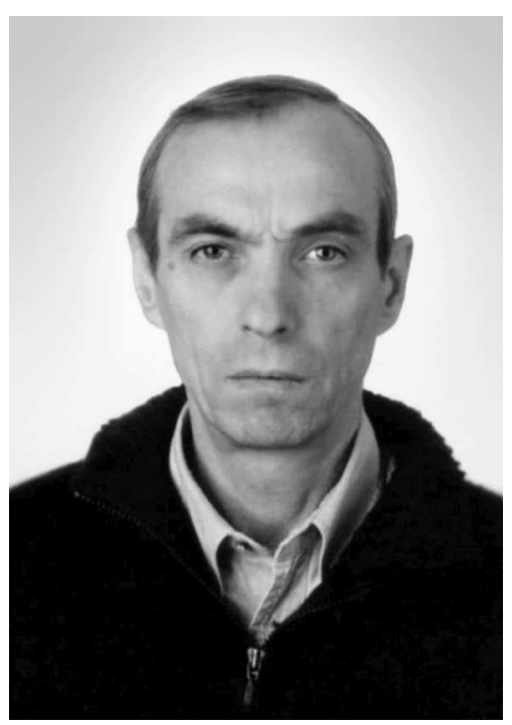
интерес к математике пробудили учителя, которых он с благодарностью вспоминал. В феврале 1963 г., десятиклассником средней общеобразовательной политехнической школы № 38 города Курска, Николай принимает участие в 4-й математической олимпиаде учащихся школ Курска и области и занимает первое место. В том же году он становится призером Всероссийской математической олимпиады и приглашается в первую летнюю математическую школу (Красновидово), организованную академиками А.Н. Колмогоровым и П. С. Александровым. По результатам зачетов его отбирают в числе 19 первых выпускников в 11-й класс физико-математической школы-интерната №18 города Москвы. В сентябре того же года он получает 3-ю премию на 2-й физико-математической олимпиаде Европейской части СССР и Закавказья, организованной сотрудниками МГУ.

В 1964 г., окончив физико-математическую школу-интернат № 18 при МГУ (преподавателями математики являлись, в частности, А. Н. Колмогоров и В. И. Арнольд, его будущий научный руководитель), Н. Н. Нехорошев поступил на механико-математический факультет МГУ. С этого времени он всю жизнь был связан с механикоматематическим факультетом. Здесь, по окончании в 1969 г. МГУ, он учился в аспирантуре на кафедре дифференциальных уравнений под руководством В. И. Арнольда, в сентябре 1973 г. защитил кандидатскую диссертацию, работал на кафедре дифференциальных уравнений, а с 2001 г. - на кафедре математического анализа. Начиная с 1972 г. Николай Николаевич совместно с Ю. С. Ильяшенко и Е. М. Ландисом руководил семинаром по обыкновенным дифференциальным уравнениям. В конце 70-х годов Ландис отошел от руководства семинаром, а семинары Нехорошева и Ильяшенко разделились. 
C 1965 г. Николай Николаевич был активным участником и организатором студенческого строительного движения на механико-математическом факультете МГУ, неоднократно выезжал с отрядами в Казахстан и на Сахалин. Он был основателем и первым командиром самого известного стройотряда мехмата - Республика Тын. Н. Н. всегда вспоминал об этом времени как о ярких страницах своей жизни.

На мехмате Николай Николаевич начал заниматься в семинаре В. И. Арнольда. В 1966 г. В.И. Арнольд, в то время молодой двадцатидевятилетний профессор мехмата, набрал группу учеников, в которую, кроме Н. Н. Нехорошева, вошли С. Здравковска, Б. М. Ивлев, А. С. Пяртли и А.Г. Хованский. Студенческие годы были насыщены интенсивным общением с научным руководителем, включающим длинные прогулки и разговоры обо всем на свете. Но, конечно, самым главным была научная работа. Николай Николаевич думал над очень трудной задачей. Работа шла нелегко. Но именно исследования, начатые Нехорошевым в студенческие годы, принесли ему впоследствии мировую известность.

Одна из первых его научных статей, написанная по материалам дипломной работы, была посвящена переменным действие-угол и их обобщениям и оказалась пионерской в теории так называемых некоммутативно интегрируемых систем, т. е. гамильтоновых систем с полной некоммутативной алгеброй первых интегралов. Такая ситуация является типичной, например, в тех случаях, когда рассматриваемая система инвариантна относительно действия некоммутативной группы Ли. Н.Н. Нехорошевым были указаны условия, при которых совместные поверхности уровня первых интегралов являются торами с квазипериодической динамикой, и доказано существование обобщенных переменных действие-угол. В случае некоммутативной алгебры первых интегралов, в отличие от случая классической интегрируемости, размерность таких торов строго меньше половины размерности фазового пространства. Впоследствии были описаны механизмы появления таких систем (иногда их называют суперинтегрируемыми) и обнаружены многочисленные примеры, в каждом из которых результат, полученный Н. Н., служит основным инструментом для описания динамики.

Кандидатская диссертация Н. Н. Нехорошева содержит доказательство замечательного и очень трудного результата об экспоненциальной медленности эволюции переменных действия в слабо возмущенных интегрируемых гамильтоновых системах. В качестве гипотезы подобные оценки рассматривал уже Дж. Литтлвуд (а возможно, и Д. Биркгоф); сегодня они являются самым сильным достижением в многомерной KAM-теории, где колмогоровские торы не делят фазовое пространство и где возможен медленный уход фазовых кривых в щелях между ними (в терминах астрономических такой уход мог бы соответствовать, скажем, падению Луны на Землю или развалу Солнечной системы; KAM-теория утверждает, что эти события маловероятны, а теория Нехорошева - что даже если они и реализуются, то крайне медленно, обычно даже и за космологические времена). Экспоненциально малая скорость накопления возмущений, доказанная Нехорошевым, только и обеспечивает длительное существование планет, астероидов и комет вблизи так называемой хаотической области. Сущность этих оценок состоит в том, что время значительного изменения параметров основного возмущаемого движения превосходит экспоненту некоторой степени обратной величины возмущения. Теория Нехорошева основана на замечательной комбинации идей теории диофантовых приближений на подмногообразиях евклидова пространства с топологическими соображениями и с оценками сумм рядов Фурье. Успеху Нехорошева способствовало выделение им важного класса невозмущенных гамильтоновых систем, названных им крутыми. Каждое аналитическое подмногообразие евклидова пространства, не лежащее ни в какой гиперплоскости, автоматически оказывается крутым. Оценки Нехорошева даются в терминах рациональных чисел, названных им показателями крутизны и характеризующих степень искривленности многообразия.

Эти работы Н.Н. Нехорошева были отмечены премией Московского математического общества (1974) и премией им. А. Н. Колмогорова РАН (1997). 
Теория Нехорошева быстро стала знаменитой, он был приглашенным докладчиком на Международном математическом конгрессе в Ванкувере в 1974 г. Эти работы Н. Н. Нехорошева сейчас являются признанным краеугольным камнем теории гамильтоновых систем, вошли в учебники и монографии. Они были продолжены многими математиками, механиками и физиками, занимавшимися приложениями теории возмущений. Многие результаты этой теории распространены (при участии Н. Н. Нехорошева) на гамильтоновы уравнения с частными производными, что также является замечательным достижением. В дальнейшем Н. Н. Нехорошев продолжал заниматься теорией гамильтоновых систем (см. ниже список публикаций). Вот формулировка одного из его результатов, оказавшегося очень полезным при исследовании солитонов. Пусть гамильтонова система имеет набор коммутирующих первых интегралов, число которых меньше числа степеней свободы. Пусть в фазовом пространстве системы имеется гладкое компактное связное многообразие размерности, равной числу интегралов, инвариантное относительно всех гамильтоновых векторных полей, порожденных этими интегралами. Тогда, при некотором условии невырожденности, это многообразие - тор, и он включается в семейство инвариантных торов, параметризуемое постоянными рассматриваемых интегралов. Взятые вместе, эти торы составляют в фазовом пространстве симплектическое подмногообразие, ограничение системы на которое становится уже обычной вполне интегрируемой системой.

Н. Н. Нехорошев участвовал в работе многих международных конференций, был приглашенным исследователем или профессором в ряде университетов. Был в Канаде, на Кубе, в Польше, США, Германии, Англии, а в последние годы - во Франции и в Италии, где он в течение трех лет был профессором Миланского университета.

Последний цикл работ Н. Н. Нехорошева был связан с исследованием обобщенной монодромии. Эта тема перекликалась с самой первой его научной работой, написанной по результатам дипломной работы. Уже тогда он обратил внимание, что простейшим препятствием к существованию глобальных переменных действие-угол является нетривиальная монодромия. Однако в то время наличие нетривиальной монодромии рассматривалось скорее как некий формальный математический факт, не имеющий интереса для физических приложений. Только значительно позже, благодаря независимой работе Х. Дюистермаата 1980 г. и усилиям Р. Кушмана, гамильтонова монодромия и ее квантовый аналог были осознаны как физические явления. Многочисленные конкретные физические, зачастую фундаментальные, задачи оказались существенным образом связанными с монодромией. Этой темой Н. Н. Нехорошев стал снова заниматься во время своего первого визита во французский университет Литтораль, в Дюнкерке. Он активно взялся за формулировку математически строгого обоснования дробной монодромии и, без сомнения, стал ключевой фигурой в построении математической теории этого явления. Его безвременная кончина оставила без решения многие вопросы дальнейшего обобщения и приложений. В мае 2008 г., полный новых идей, Н.Н. Нехорошев снова собирался в Дюнкерк. Однако за неделю до отъезда поездку пришлось отменить в связи с резким неожиданным ухудшением здоровья. Далеко идущие планы не смогли реализоваться.

А.М. Абрамов, В.И. Арнольд, А.В. Болсинов, А.Н. Варченко, Л. Гальгани, Б. И. Жилинский, Ю. С. Ильяшенко, В. В. Козлов, А. И. Нейштадт, В.И. Питербарг, А.Г. Хованский, В.В. Ященко

\section{Список основных научных трудов Н. Н. Нехорошева}

[1] “Две теоремы о переменных действие-угол”, УМН, 24:5 (1969), 237-238.

[2] "О поведении гамильтоновых систем, близких к интегрируемым", Функи. анализ и его прил., 5:4 (1971), 82-83; англ. пер.: "Behavior of Hamiltonian systems close to integrable", Funct. Anal. Appl., 5:4 (1971), 338-339. 
[3] "Переменные действие-угол и их обобщение", Тp. MMO, 26 (1972), 181-198; англ. пер.: "Action-angle variables, and their generalizations", Trans. Moscow Math. Soc., 26 (1974), 180-198.

[4] "Устойчивые оценки снизу для гладких отображений и для градиентов гладких функций", Матем. сб., 90:3 (1973), 432-478; англ. пер.: "Stable lower estimates for smooth mappings and for gradients of smooth functions", Math. USSR-Sb., 19:3 (1973), 425-467.

[5] О поведении гамильтоновых систем, близких к интегрируемым, Дис. ... канд. физ.-матем. наук, МГУ, М., 1973.

[6] "Метод последовательных канонических замен переменных", Добавление к кн.: Ю. Мозер, Лекиии о гамильтоновых системах, Мир, М., 1973, 150-164.

[7] "О поведении гамильтоновых систем, близких к интегрируемым", Proceedings of the International Congress of Mathematicians (Vancouver, BC, 1974), 2, Canad. Math. Congress, Montreal, Quebec, 1975, 309-314.

[8] "Экспоненциальная оценка времени устойчивости гамильтоновых систем, близких к интегрируемым", УМН, 31:3 (1976), 195.

[9] “Экспоненциальная оценка времени устойчивости гамильтоновых систем, близких к интегрируемым", УМH, 32:6 (1977), 5-66; англ. пер.: "An exponential estimate of the time of stability of nearly-integrable Hamiltonian systems", Russian Math. Surveys, 32:6 (1977), 1-65.

[10] “Экспоненциальная оценка времени устойчивости гамильтоновых систем, близких к интегрируемым. II", Тр. сем. им. И. Г. Петровского, 5 (1979), 5-50; англ. пер.: "An exponential estimate of the time of stability of nearly integrable Hamiltonian systems. II", Topics in modern mathematics, Petrovskii Semin., 5 (1985), 1-58.

[11] "Нормальные формы неприводимых особенностей функций от двух переменных", УMH, 36:4 (1981), 234.

[12] "Факторы, влияющие на устойчивость гамильтоновых систем, близких к интегрируемым”, IX-я международная конферениия по нелинейным колебаниям, т. 2 (Киев, 1981), Наукова думка, Киев, 1984, 292-294.

[13] "О примыкании особенностей типа $A_{k}$ к страту $\mu=$ const", УMH, 38:5 (1983), 127 (совм. с С. М. Гусейн-Заде).

[14] "О примыканиях особенностей $A_{k}$ к точкам страта $\mu=$ const особенности", Функи. анализ и его прил., 17:4 (1983), 82-83 (совм. с С. М. Гусейн-Заде); англ. пер.: "Contiguity of $A_{k}$-singularities at points of the stratum $\mu=$ const of a singularity", Funct. Anal. Appl., 17:4 (1983), 312-313 (with S. M. Gusein-Zade).

[15] "Комплексный росток в системах с одной циклической переменной", УМH, 39:3 (1984), 233-234 (совм. с Б. Валиньо, С. Ю. Доброхотовым); англ. пер.: "Тhе complex germ in systems with one cyclic variable", Russian Math. Surveys, 39:3 (1984), 219-220 (with B. Valigno, S. Yu. Dobrokhotov).

[16] "Условия существования и единственности комплексного ростка на изотропных торах", УМН, 39:4 (1984), 124 (совм. с Б. Валиньо).

[17] “Об одной теореме Н. И. Гаврилова”, Дифферени. уравнения, 21:2 (1985), 335-338.

[18] "Семейства инвариантных изотропных торов гамильтоновых систем”, УМН, 40:5 (1985), 217.

[19] "Ряды Пюизе от двух переменных", УМН, 44:4 (1989), 225.

[20] "Сильно устойчивые периодические траектории в резонансных зонах максимальной кратности”, УМН, $45: 4$ (1990), 116.

[21] "Superstability of the systems with quasi-convex unperturbed Hamiltonians", 1991.

[22] "Stability of the resonant zones of the systems with quasi-convex unperturbed Hamiltonians", 1991. 
[23] "Резонансные зоны многочастотных систем", УМH, 46:6 (1991), 178; англ. пер.: "Resonance zones of multifrequency systems", Russian Math. Surveys, 46:6 (1991), 190-191.

[24] "Теорема Пуанкаре-Ляпунова-Лиувилля-Арнольда", Функи. анализ и его прил., 28:2 (1994), 67-69; англ. пер.: "The Poincaré-Lyapunov-Liouville-Arnol'd theorem", Funct. Anal. Appl., 28:2 (1994), 128-129.

[25] "О комплексных структурах на двумерных торах, допускающих метрики с нетривиальным квадратичным интегралом", Матем. заметки, 58:5 (1995), 643-652 (совм. с И.КК. Бабенко); англ. пер.: "On complex structures on two-dimensional tori admitting metrics with nontrivial quadratic integral", Math. Notes, 58:5 (1995), 1129-1135 (with I. K. Babenko).

[26] "A property of exponential stability in nonlinear wave equations near the fundamental linear mode", Phys. D, 122:1-4 (1998), 73-104 (with D. Bambusi).

[27] "Оценка числа выживающих при возмущении замкнутых траекторий гамильтоновых систем", УМН, 53:4 (1998), 206-207 (совм. с Е. А. Кудрявцевой); англ. пер.: "A bound for the number of closed trajectories of Hamiltonian systems viable under perturbation", Russian Math. Surveys, 53:4 (1998), 857 (with E. A. Kudryavtseva).

[28] “Экспоненциальная устойчивость приближенной основной моды нелинейного волнового уравнения", Функи. анализ и его прил., 33:1 (1999), 80-83; англ. пер.: "Exponential stability of the approximate principal mode of the nonlinear wave equation", Funct. Anal. Appl., 33:1 (1999), 69-71.

[29] "Об особенностях типа $A_{k}$ на плоских кривых фиксированной степени", Функи. анализ и его прил., 34:3 (2000), 69-70 (совм. с С. М. Гусейн-Заде); англ. пер.: "Singularities of type $A_{k}$ on plane curves of a chosen degree", Funct. Anal. Appl., 34:3 (2000), 214-215 (with S. M. Gusein-Zade); arXiv: math/9906147

[30] "Generalizations of Gordon theorem", Regul. Chaotic Dyn., 7:3 (2002), 239-247.

[31] "Сильная устойчивость приближенной основной моды нелинейного уравнения струны", Тр. MMO, 63 (2002), 166-236; англ. пер.: "Strong stability of an approximate fundamental mode of a nonlinear string equation", Trans. Moscow Math. Soc., 2002, 151-217.

[32] "Long time stability in perturbations of completely resonant PDE's", Act. Appl. Math., 70:1-3 (2002), 1-22 (with D. Bambusi).

[33] "Fractional monodromy of resonant classical and quantum oscillators", C. R. Math. Acad. Sci. Paris, 335:11 (2002), 985-988 (with D. A. Sadovskii, B. I. Zhilinskii).

[34] “Типы интегрируемости на подмногообразии и обобщения теоремы Гордона", Tp. MMO, 66 (2005), 184-262; англ. пер.: "Types of integrability on a submanifold and generalizations of Gordon's theorem", Trans. Moscow Math. Soc., 2005, 169-241.

[35] "Fractional Hamiltonian monodromy", Ann. Henri Poincaré, 7:6 (2006), 1099-1211 (with D. A. Sadovskií, B. I. Zhilinskií).

[36] “Дробная монодромия в случае произвольных резонансов", Матем. сб., 198:3 (2007), 91-136; англ. пер.: "Fractional monodromy in the case of arbitrary resonances", Sb. Math., 198:3 (2007), 383-424.

[37] N. N. Nekhoroshev, "Fuzzy fractional monodromy and the section-hyperboloid", $M i$ lan J. Math., 76:1 (2008), 1-14.

[38] Integrable quantum systems obtained by quantization, Preprint, Milan, 2008 (with M. Marino).

[39] A Liouville theorem for contact manifolds, Preprint, Milan, 2008 (with L. Pizzocchero).

[40] Монодромия слоя с осцилляторной особой точкой типа 1:(-2), рукопись, 2008. 